\title{
Trypsin inhibition by macrocyclic and open-chain variants of the squash inhibitor MCoTI-II
}

\author{
Olga Avrutina1, Hans-Ulrich Schmoldt², Dusica \\ Gabrijelcic-Geiger ${ }^{3}$, Dung Le Nguyen ${ }^{4}$, \\ Christian P. Sommerhoff ${ }^{3}$, Ulf Diederichsen ${ }^{1}$ \\ and Harald Kolmar ${ }^{2, *}$ \\ ${ }^{1}$ Institute for Organic and Biomolecular Chemistry, \\ Georg-August-University, D-37077 Göttingen, Germany \\ ${ }^{2}$ Department of Molecular Genetics, Institute for \\ Microbiology and Genetics, Georg-August-University, \\ D-37077 Göttingen, Germany \\ ${ }^{3}$ Department Clinical Chemistry and Clinical \\ Biochemistry, Ludwig-Maximilians-University, D-80336 \\ Munich, Germany \\ ${ }^{4}$ Centre de Pharmacologie et Biotechnologie pour la \\ Santé, CNRS UMR 5160, 15 avenue Charles Flahault, \\ F-34093 Montpellier Cedex 5, France \\ ${ }^{*}$ Corresponding author \\ e-mail: hkolmar@gwdg.de
}

\begin{abstract}
MCoTI-I and MCoTI-II from the seeds of Momordica cochinchinensis are inhibitors of trypsin-like proteases and the only known members of the large family of squash inhibitors that are cyclic and contain an additional loop connecting the amino- and the carboxy-terminus. To investigate the contribution of macrocycle formation to biological activity, we synthesized a set of open-chain variants of MCoTI-II that lack the cyclization loop and contain various natural and non-natural amino acid substitutions in the reactive-site loop. Upon replacement of $\mathrm{P} 1$ lysine residue \#10 within the open-chain variant of MCoTI-II by the non-natural isosteric nucleo amino acid AlaG [ $\beta$-(guanin-9-yl)-L-alanine], a conformationally restricted arginine mimetic, residual inhibitory activity was detected, albeit reduced by four orders of magnitude. While the cyclic inhibitors MCoTI-I and MCoTI-II were found to be very potent trypsin inhibitors, with picomolar inhibition constants, the open-chain variants displayed an approximately 10-fold lower affinity. These data suggest that the formation of a circular backbone in the MCoTI squash inhibitors results in enhanced affinity and therefore is a determinant of biological activity.
\end{abstract}

Keywords: cyclic squash inhibitors; cystine-knot microproteins; nucleic amino acids; trypsin inhibition.

\section{Introduction}

MCoTI-I and MCoTI-II, natural polypeptides isolated from the seeds of the spinal gourd Momordica cochinchinensis, are plant proteins that have a cyclic backbone and a knotted arrangement of three conserved disulfide bonds (Hernandez et al., 2000). The cystine knot (CK) is defined by three intramolecular disulfide bonds, where CyslCysIV and Cysll-CysV of the linear peptide sequence form a ring that is penetrated by the third disulfide bond, Cyslll-CysVI (Figure 1A). This arrangement provides a well-defined and extremely stable scaffold that exhibits extraordinary thermal and proteolytic stability (Craik, 2001). Cyclic cystine-knot peptides (cyclotides) have also been found in Rubicaea and Violaceae, where they have a wide range of bioactivities, from insecticidal (Jennings et al., 2001), antimicrobial (Tam et al., 1999), and anti-HIV functions (Gustafson et al., 2004) to neurotensin binding (Witherup et al., 1994) and hemolytic activity (Daly et al., 1999), suggesting potential therapeutic and agricultural applications (Trabi and Craik, 2002).

Due to structural similarity and common biological activity, i.e., inhibition of proteases of the trypsin family, MCoTI-I and MCoTI-II have been grouped into the squash inhibitor cystine-knot (ICK) family of small protease inhibitors. Members of this family are open-chain molecules forming a small triple-stranded $\beta$-sheet and a short $3_{10}$ helix, held together by three intramolecular disulfide bonds to give rise to a cystine-knot framework. Comparison of the backbone conformation of the openchain trypsin inhibitors EETI-II (Favel et al., 1989) and CMTI (Bode et al., 1989) with the cyclic inhibitor MCoTIII (Hernandez et al., 2000; Felizmenio-Quimio et al., 2001) reveals extensive structural similarities among these molecules.

The contribution of the head-to-tail cyclization of the backbone to overall microprotein stability and rigidity is currently not well defined. For the cyclotide kalata B1, both a loss of biological activity and a disruption of some structural features that are important to the overall stability were observed when the macrocycle was opened and five residues removed (Barry et al., 2003). However, it appears that the cystine knot is more important than the circular backbone for the chemical stability of the cyclotide (Colgrave and Craik, 2004).

Interestingly, in the same natural source of $M$. cochinchinensis, both cyclic and open-chain variants of cystine knot peptides are found (Hernandez et al., 2000; Figure $1 \mathrm{~B})$. It is unclear whether the additional loop sequence that connects the amino- and the carboxy-terminus contributes to overall stability, framework rigidity, resistance against proteolytic degradation, or inhibitory activity. To investigate whether and how backbone cyclization influences the biological activity of MCoTI-II, i.e., inhibition of trypsin and trypsin-like proteases, we compared trypsin inhibition of the cyclotides MCoTI-I and MCoTI-II with the open-chain variant MCoTI-III and a set of chemically synthesized open-chain variants of MCoTI-II with amino acid substitutions within the reactive site loop. 
A

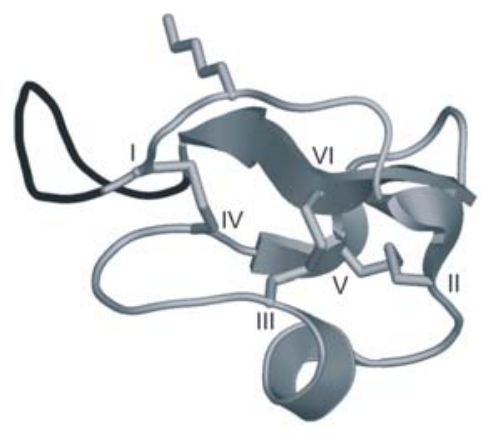

B

MCOTI-I: CYC.-SGSDGGVC PKILQRC $C^{11}$ RRDSDC $^{\text {III PGAC }} C^{I V} \mathrm{VC}^{\mathrm{V}}$ RGNGYC ${ }^{\mathrm{VI}} \mathrm{G}$

MCOTI-II: cyc.-SGSDGGVC ${ }^{1}$ PKILKKC ${ }^{11}$ RRDSDC $^{I I I}$ PGAC $^{I V} \mathrm{VC}^{\mathrm{V}}$ RGNGYC ${ }^{\mathrm{VI}} \mathrm{G}$

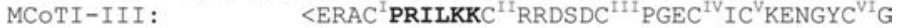

Figure 1 Trypsin inhibitors from $M$. cochinchinensis.

(A) Schematic representation of the three-dimensional structure of the cyclic trypsin inhibitor McoTI-II (Heitz et al., 2001). Secondary structure elements are indicated. The disulfide bonds forming the cystine knot architecture and the side chain of the P1 residue lysine \#10 are indicated as sticks. The macrocycle-forming loop is shown in black. (B) Amino acid sequences of the naturally occurring microproteins MCoTI-I, MCoTI-II, and MCoTI-III. Amino acids of the inhibitor loop are indicated in bold letters. $<$ E, amino-terminal pyro-Glu of MCoTI-III (Hernandez et al., 2000).

\section{Results and discussion}

The aim of this study was to elucidate whether backbone cyclization in the squash inhibitor MCoTI-II, which was isolated from seeds of $M$. cochinchinensis by virtue of its ability to inhibit trypsin (Hernandez et al., 2000; Chiche et al., 2004), contributes to its biological activity. A set of open-chain variants of MCoTI-II was synthesized via 9-fluorenylmethoxycarbonyl (Fmoc) synthesis, as described recently (Avrutina et al., 2004), and product identities were confirmed by ESI mass spectroscopy (Table 1). Open-chain MCoTI-II (oMCoTI-II) differs from the natural cyclic MCoTI-II only in that it lacks part of the cyclization loop (amino acid residues 1-5; Figure 1B). This loop sequence is also absent in MCoTI-III and not found in related open-chain trypsin inhibitors from other plants of the squash family, e.g., CMTI-I (Bode et al., 1989) and EETI-II (Favel et al., 1989).

\section{Contribution of basic residues within the reactive site loop to trypsin inhibition}

oMCoTI-II is a strongly basic microprotein (calculated pl 8.9), in which three lysine residues are located within the reactive site loop and two arginines in tandem are directly adjacent to it. To investigate the contribution of the basic residues within the inhibitor loop to trypsin binding, an alanine scanning of $\mathrm{K} 10, \mathrm{~K} 13$, and $\mathrm{K} 14$ of oMCoTI-II was performed, together with an individual replacement of these residues by arginines (Figure 2). As expected from comparison of the MCoTI-II structure (Heitz et al., 2001) with structural data of closely related open-chain squash inhibitors in complex with trypsin (Bode et al., 1989), the role of lysine \#10 of oMCoTI-Il as the active-site P1 residue and primary determinant of inhibitor specificity (Schechter and Berger, 1967) was confirmed, since replacement by alanine completely abolished trypsin inhibition (Table 1). In contrast, substitution of lysines \#13 and \#14 by alanine or arginine affected the affinity towards trypsin only slightly, suggesting that these inhibitor residues contribute little to protease binding.

\section{Replacement of the oMCoTI-II P1 residue by the non-natural amino acid AlaG}

We recently described the synthesis of an oMCoTI-II variant that contains the non-natural amino acid AlaG (Figure 3 ) in place of $P 1$ residue $K 10$. AlaG is a peptide nucleic acid that contains a guanine in its side chain and is an isosteric, conformationally restricted arginine mimetic with reduced basicity of the guanidino group (Lorenz and Diederichsen, 2003) and additional proton donor and acceptor functionalities. Previous experiments (Domingo et al., 1995) with synthetic mimetics of the Bowman-Birk inhibitor containing sets of natural and non-natural amino acids at the P1 position showed that only Arg- and Lys-containing peptides were able to bind to trypsin and to inhibit it. It was concluded that trypsin inhibition depends not only on the basicity of the $\mathrm{P} 1$ residue, but also on steric factors, since a peptide containing ornithine, an amino acid that differs from lysine only by a single methylene group, had no measurable trypsin inhibitory activity (Domingo et al., 1995). In light of these previous findings, it is interesting to note that oMCoTIAlaG retains measurable residual trypsin inhibitory activity, albeit approximately four orders of magnitude lower compared to oMCoTI-II and oMCoTI-IIR10, corroborating the ability of non-natural arginine analogues to act as basic $\mathrm{P} 1$ residues in serine protease inhibitors (Kokko et al., 2001; McBride and Leatherbarrow, 2001). To understand the influence of steric effects of the AlaG side chain on trypsin binding and inhibition, structural information is required. It may therefore be interesting to see whether it will be possible to obtain a crystal structure of the complex of trypsin with oMCoTI-II or oMCoTI-AlaG. 
Table 1 List of the open-chain and cyclic cystine-knot microproteins studied.

\begin{tabular}{|c|c|c|c|c|c|}
\hline \multirow[t]{2}{*}{$\sim$} & \multirow[t]{2}{*}{ Peptide } & \multirow{2}{*}{$\begin{array}{l}\text { Sequence of } \\
\text { inhibitor loop }\end{array}$} & \multicolumn{2}{|c|}{ ESI-MS (m/z) } & \multirow{2}{*}{$\begin{array}{c}K_{\mathrm{i}} \\
(\mathrm{nM})\end{array}$} \\
\hline & & & Calculated & Measured & \\
\hline 1 & oMCoTI-II & -PKILKK- & 3066.7 & $\begin{array}{l}767.78(\mathrm{M}+4 \mathrm{H})^{4+} \\
1023.38(\mathrm{M}+3 \mathrm{H})^{3+} \\
1030.4(\mathrm{M}+2 \mathrm{H}+\mathrm{Na})^{3+}\end{array}$ & 0.3 \\
\hline 2 & oMCoTI-IIA10 & -PAILKK- & 3010.6 & $\begin{array}{l}1004.1(\mathrm{M}+3 \mathrm{H})^{3+} \\
1506.0(\mathrm{M}+2 \mathrm{H})^{2+}\end{array}$ & $\mathrm{NI}$ \\
\hline 3 & oMCoTI-IIA13 & -PKILAK- & 3010.6 & $\begin{array}{l}753.4(\mathrm{M}+4 \mathrm{H})^{4+} \\
1004.1(\mathrm{M}+3 \mathrm{H})^{3+} \\
1506.0(\mathrm{M}+2 \mathrm{H})^{2+}\end{array}$ & 0.2 \\
\hline 4 & oMCoTI-IIA14 & PKILKA- & 3010.6 & $\begin{array}{l}753.6(\mathrm{M}+4 \mathrm{H})^{4+} \\
1004.1(\mathrm{M}+3 \mathrm{H})^{3+} \\
1505.8(\mathrm{M}+2 \mathrm{H})^{2+}\end{array}$ & 0.4 \\
\hline 5 & oMCoTI-IIR10 & -PRILKK- & 3094.7 & $\begin{array}{l}620.1(\mathrm{M}+5 \mathrm{H})^{5+} \\
774.8(\mathrm{M}+4 \mathrm{H})^{4+} \\
1039.8(\mathrm{M}+2 \mathrm{H}+\mathrm{Na})^{3+}\end{array}$ & 0.8 \\
\hline 6 & oMCoTI-IIR13 & -PKILRK- & 3094.7 & $\begin{array}{l}620.1(\mathrm{M}+5 \mathrm{H})^{5+} \\
774.8(\mathrm{M}+4 \mathrm{H})^{4+} \\
780.2(\mathrm{M}+3 \mathrm{H}+\mathrm{Na})^{4+} \\
1032.4(\mathrm{M}+3 \mathrm{H})^{3+} \\
1039.8(\mathrm{M}+2 \mathrm{H}+\mathrm{Na})^{3+}\end{array}$ & 0.5 \\
\hline 7 & oMCoTI-IIR14 & -PKILKR- & 3094.7 & $\begin{array}{l}774.9(\mathrm{M}+4 \mathrm{H})^{4^{+}} \\
1032.5(\mathrm{M}+3 \mathrm{H})^{3+}\end{array}$ & 0.4 \\
\hline 8 & oMCoTI-IIAlaG10 & -PAlaGILKK- & 3158.7 & $\begin{array}{l}790.7(\mathrm{M}+4 \mathrm{H})^{4+} \\
1054.0(\mathrm{M}+3 \mathrm{H})^{3+} \\
1580.7(\mathrm{M}+2 \mathrm{H})^{2+}\end{array}$ & 1000 \\
\hline 9 & MCoTI-I & -PKILQR- & 3480.96 & $\begin{array}{l}870.62280(\mathrm{M}+4 \mathrm{H})^{4+} \\
696.69980(\mathrm{M}+5 \mathrm{H})^{5+}\end{array}$ & 0.02 \\
\hline 10 & MCoTI-II & -PKILKK- & 3452.99 & $\begin{array}{l}863.63055(\mathrm{M}+4 \mathrm{H})^{4+} \\
691.10583(\mathrm{M}+5 \mathrm{H})^{5+}\end{array}$ & 0.03 \\
\hline 11 & MCoTI-III & -PRILKK- & 3379.6 & $\begin{array}{l}845.37633(\mathrm{M}+4 \mathrm{H})^{4+} \\
676.50259(\mathrm{M}+5 \mathrm{H})^{5+}\end{array}$ & 0.5 \\
\hline
\end{tabular}

1-8 are synthetic open-chain peptides and 9-11 are natural microproteins from Momordica cochinchinensis. The amino acid sequences of the respective inhibitor loops are indicated, as well as ESI MS data and inhibition constants against bovine trypsin. Bold letters indicate amino acid substitutions. AlaG, peptide nucleic acid with guanidino function in the side chain. Numbering of amino acids is according to Hernandez et al. (2000). NI, no inhibition detected at a protein concentration of $10 \mu \mathrm{M}$.

\section{Trypsin inhibition of cyclic inhibitors compared to open-chain variants}

The inhibition of trypsin by the open-chain variants of MCoTI-II was compared with that caused by MCoTI-II isolated from squash seeds and by the chemically synthesized cyclic MCoTI-I and open-chain MCoTI-III (Figure 4). With an equilibrium dissociation constant $K_{\mathrm{i}}$ of $0.3 \mathrm{~nm}$ (Table 1) the affinity of oMCoTI-II towards trypsin is lower than that of other open-chain CKIs from squash such as EETI-II (Favel et al., 1989; $K_{\mathrm{i}}=0.08 \mathrm{nM}$ ). However, cyclic MCoTI-I and MCoTI-II are more potent inhibitors, with $K_{\mathrm{i}}$ values of 0.02 and $0.03 \mathrm{nM}$, respectively, while openchain MCoTI-III $(0.5 \mathrm{nM})$ is comparable to open-chain oMCoTI-II. These data show that the cyclic feature of MCoTI-I and MCoTI-II contributes to the affinity towards trypsin.

What is the reason for the differences in trypsin inhibition between the macrocyclic trypsin inhibitors and the variants lacking a C-to-N cyclization? One possibility would be that the cyclization loop (SGSDGG) provides additional binding energy via direct interaction with trypsin that contributes to enhanced binding and inhibition. Unfortunately, the structure of a complex of a cyclic squash inhibitor with trypsin has not yet been described, and structural data of the open-chain variants are also not available. To determine whether any of the six cyclization-loop residues could contribute to trypsin binding, we used the structures of the complex of the squash inhibitor EETI-II with porcine trypsin (Krätzner et al., 2005) and of free MCoTI-II (Heitz et al., 2001) and of bovine trypsin as templates to model the interaction of this microprotein with trypsin (Figure 5). According to the model, none of the trypsin residues comes into proximity to the connecting loop closer than $5.0 \AA$. Only valine \#7, which is directly adjacent to the connecting loop and is present in our open-chain variants of MCoTI-II, is close to trypsin residues, namely tryptophan \#215 and tyrosine \#175 (2.1 and $2.6 \AA$, respectively) These two residues are good candidates to provide binding energy by hydrophobic interactions with the side chain of valine \#7, which may be in a position more favorable for trypsin interaction when placed adjacent to the conformationally constrained cyclization loop of MCoTI-II. Notably, alanine and not valine is present in MCoTI-III at this position (Figure 1) and replacement of valine \#7 by alanine both in cyclic and open-chain MCoTI-II could clarify whether this residue significantly contributes to trypsin binding. More- 


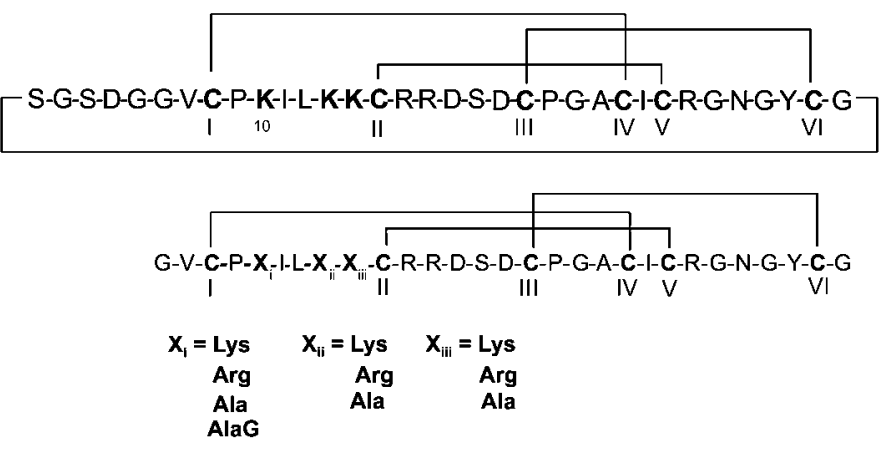

Figure 2 Amino acid sequence and disulfide bond connectivity of the cyclotide MCoTI-II (upper panel) and the open-chain variants described in this study (lower panel).

over, other indirect contributions of the connecting loop to enhanced trypsin inhibition appear possible. For example, $\mathrm{N}$-to-C macrocycle formation is likely to introduce additional conformational rigidity into the already constrained molecule, which subsequently could contribute to a better fit of the inhibitor loop into the active site of the enzyme.

\section{Conclusion}

In the current study we determined the affinity of the natural cyclic CKIs MCoTI-I and MCoTI-II towards trypsin compared with the natural open-chain variant MCoTI-III and a series of MCoTI-Il derivatives lacking the macrocycle-forming loop. Alanine scanning mutagenesis suggests that MCoTI-II binds, similar to other inhibitors of the squash family, the target enzyme in a substrate-like manner, with lysine \#10 in the inhibitor loop acting as P1 residue and determinant of selectivity, while the side chains of the other two basic residues in the inhibitor loop contribute to trypsin binding only to a small extent.

Compared to the cyclic inhibitors MCoTI-I and MCoTIII that are very potent trypsin inhibitors, the affinity of the open-chain variants is approximately 10 -fold lower. More structural information on the cyclic and open-chain forms of MCoTI-II in solution and in complex with trypsin is required to clarify why the cyclic squash inhibitor MCoTIII is a better trypsin inhibitor than its open-chain counterpart.<smiles>CN[C@@H](CCCNC(=N)N)C(C)=O</smiles>
1<smiles>[Z]N[C@@H](Cn1cnc2c(=O)[nH]c(N)nc21)C(C)=O</smiles>

2
Figure 3 Nucleo amino acid AlaG (2) as an isoster for Arg (1). The isosteric relationship between Arg and AlaG is marked with highlighted bonds.

\section{Materials and methods}

\section{Materials}

All chemicals used were of the highest grade available. Solvents were of analytical grade and used as supplied. Fmoc-protected amino acids were used with the following side-chain protecting groups: tert-butyl (Asp, Tyr), tert-butoxycarbonyl (Lys), trityl (Cys, Asn), 2,2,4,6,7-pentamethyldihydro-benzofuran-5-sulfonyl (Arg). Pseudo-proline dipeptide Fmoc-Asp(OtBu)-Ser $\left(\psi^{\mathrm{Me}, \mathrm{Me}}\right)$ pro-OH was purchased from Calbiochem-Novabiochem GmbH (Schwalbach, Germany). Electrospray ionization (ESI) mass spectra were measured with a Finnegan (San Jose, USA) LCQ spectrometer. High-resolution ESI mass spectra were recorded with a Bruker APEX-Q III 7T. High-performance liquid chromatography (HPLC) was performed with a Pharmacia (Freiburg, Germany) Äcta basic system using YMC (Dinslaken, Germany) J'sphere ODS H-80, RP C-18 $(250 \times 20 \mathrm{~mm}, 4 \mu \mathrm{m}, 8 \mathrm{~nm})$ and Phenomenex (Aschaffenburg, Germany) Synergi 4u Hydro-RP 80A $(250 \times 10 \mathrm{~mm}$, $4 \mu \mathrm{m}, 8 \mathrm{~nm})$ columns for preparative separations and YMC J'sphere ODS H-80, RP C-18 (250×4.6 mm, $4 \mu \mathrm{m}, 8 \mathrm{~nm})$ and Phenomenex Synergi 4u Hydro-RP 80A (250×4.6 mm, $4 \mu \mathrm{m}$, $8 \mathrm{~nm}$ ) for analytical samples.

\section{Reference microproteins: MCoTI-I, MCoTI-II and MCoTI-III}

Cyclotide MCoTI-II was isolated from the seeds of $M$. cochinchinensis (Hernandez et al., 2000). Cyclic MCoTI-I and openchain MCoTI-III were synthesized on a solid support. Details on the preparation of synthetic peptides will be published elsewhere.

\section{Synthesis and oxidation of oMCoTI-II peptides}

Peptide synthesis was conducted using Fmoc solid-phase peptide synthesis (SPPS) on PEG-type amide NovaSyn ${ }^{\circledR}$ TGR resin (Calbiochem-NovaBiochem) with a loading capacity of 0.23 $\mathrm{mmol} / \mathrm{g}$ and involved several steps. After manual attachment of the C-terminal glycine as previously described (Avrutina et al., 2004), the linear precursor was assembled up to $C 10$ using the automated mode on an $\mathrm{ABI} 433$ peptide synthesizer. The conditions for the deprotection, activation, and coupling of all amino acids except cysteine were the same as described by Avrutina et al. (2004). For the self-automated incorporation of cysteine, an additional program was created to provide conditions for racemization-free coupling: N,N-diisopropyl carbodiimide (DIC)/ 1-hydroxy-1H-benzotriazole (HOBt) activation with 10-min preactivation and coupling time of $1 \mathrm{~h} 20 \mathrm{~min}$. DIPEA delivery was removed from the activation cycle by manual programming. 


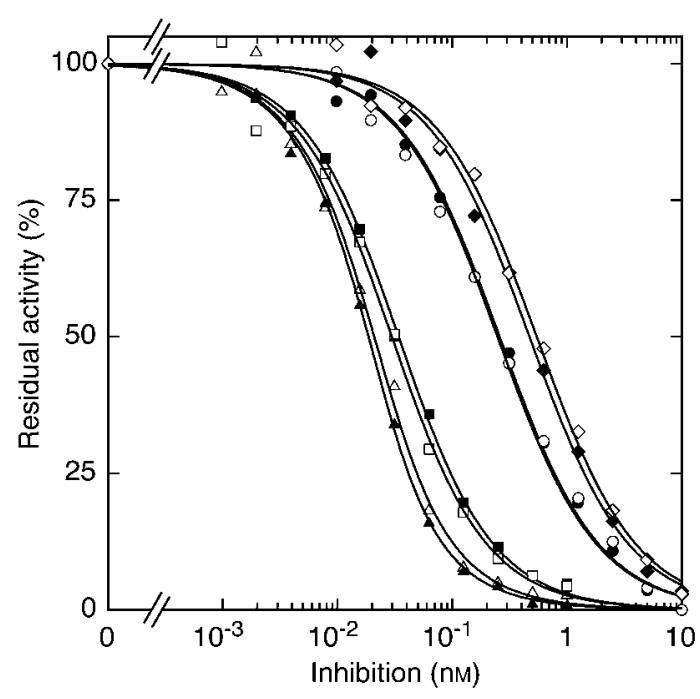

Figure 4 Inhibition of trypsin by the naturally occurring cyclic MCoTI-I (triangles) and MCoTI-II (squares), the open-chain MCoTI-III (diamonds), and the synthetic open-chain variant oMCoTI-II (circles).

For each inhibitor, two inhibition curves are shown that were generated by fitting data derived from separate experiments (open and filled symbols) to Eq. (1), as described in the materials and methods section.

HOBt was added as a solid directly onto the amino acid cartridge according to a standard coupling protocol (Albericio et al., 2000), and DIC was used as a $5 \%$ solution in dichloromethane. To achieve practical DIC addition, methanol from the bottle at position 5 was replaced with an activator solution, and the time was regulated to deliver $2 \mathrm{ml}$ of $5 \%$ DIC into the cartridge.

After automated peptide synthesis, the resin was dried and a small portion was subjected to a test cleavage to assess the quality of the peptide. Then portions of $100 \mathrm{mg}$ each were placed into polypropylene syringes, and the synthesis was continued manually to obtain different resin-bound polypeptides with altered inhibitor-loop sequences.

The peptide resins were dried for $24 \mathrm{~h}$ and the peptides were then cleaved off by treatment with trifluoroacetic acid, purified by HPLC, air-oxidized and re-purified by HPLC to give folded microproteins (Avrutina et al., 2004; Table 1).

\section{Enzymatic methods}

The concentrations of the inhibitory active microproteins were determined by titration with trypsin. Therefore, bovine pancreatic trypsin $(\mathrm{N}-\mathrm{p}$-tosyl-L-phenylalanine chloromethyl ketone-treated; Sigma, Munich, Germany) was standardized by active-site titration using $p$-nitrophenyl- $p^{\prime}$-guanidinobenzoate (Chase and Shaw, 1970). Trypsin (25 nM) was then incubated with serial dilutions of an inhibitor in buffer $(50 \mathrm{mM}$ Tris/ $\mathrm{HCl}, 150 \mathrm{mM} \mathrm{NaCl}$, $0.01 \%$ Triton $\mathrm{X}-100,0.01 \%$ sodium azide, $\mathrm{pH} 7.6)$. After $30 \mathrm{~min}$, carbobenzoxy-L-arginine-7-amino-4-methylcoumarin (75 $\mu \mathrm{M}$; Sigma) was added, and the residual activity was quantified by following the hydrolysis of the substrate over $10 \mathrm{~min}$. Measurements were performed in 96-well plates (Corning, Corning, USA) at room temperature using a HTS 7000 Bio Assay Reader (Perkin Elmer, Rodgau-Jügesheim, Germany) with excitation and emission wavelengths of 360 and $465 \mathrm{~nm}$, respectively. The concentration of the active inhibitor was calculated from inhibition curves obtained in two separate experiments assuming a 1:1 interaction between inhibitor and trypsin.

Equilibrium dissociation constants $\left(K_{i}\right)$ for the complexes of the inhibitors with trypsin were determined essentially as described above, but using an enzyme concentration in the range of or lower than $K_{\mathrm{i}}$ (Bieth, 1980). Thus, trypsin (0.005 or $0.6 \mathrm{nM}$ ) was incubated with serial dilutions of an inhibitor in buffer for 30-90 min; the time required to reach equilibrium was verified in separate experiments. The residual trypsin activity was then measured following the breakdown of $5 \mu \mathrm{MN} N$-p-tosylglycine-proline-arginine-7-amido-4-methylcoumarin (Sigma) or $75 \mu \mathrm{M}$ carbobenzoxy-L-arginine-7-amino-4-methylcoumarin. The data were fitted to Eq. (1) (Morrison, 1969) using a Levenberg-Marquardt algorithm with proFit (Quantum Soft, Uetikon am See, Switzerland):

$v_{\mathrm{i}}=v_{0}\left(1-\frac{\left(E_{0}+I_{0}+K\right)-\sqrt{\left(E_{0}+I_{0}+K\right)^{2}-4 E_{0} I_{0}}}{2 E_{0}}\right)$

where $v_{0}$ and $v_{i}$ are the enzyme activity measured in the absence and presence of the inhibitor, and $E_{0}$ and $I_{0}$ the total enzyme and inhibitor concentrations, respectively. Under the experimental conditions used, addition of substrate does not cause significant dissociation of the enzyme-inhibitor complex, so that $K=K_{\mathrm{i}} . K_{\mathrm{i}}$ values determined in separate experiments differed by less than two-fold for all inhibitors, and averages of at least two experiments are reported.
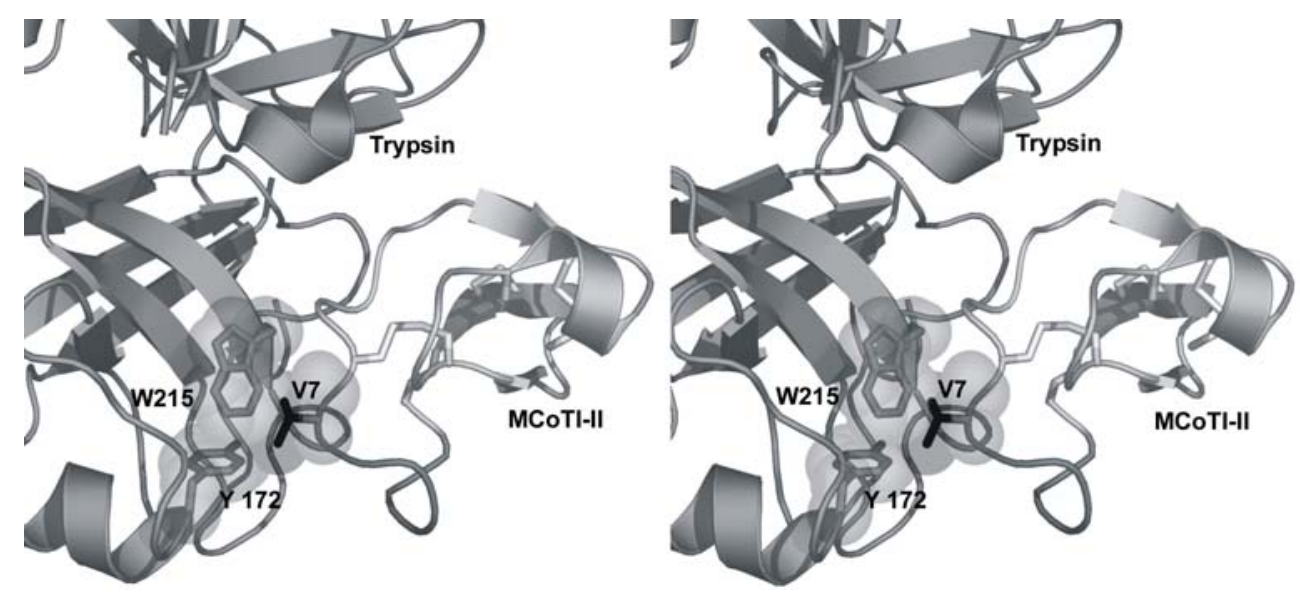

Figure 5 Model of the complex of MCoTI-II with trypsin.

The structure of MCOTI-II (PDB entry 1HA9) was superimposed onto the structure of the squash inhibitor EETI-II in complex with porcine trypsin (see materials and methods). The side chains of the trypsin residues Y172 and W215 that are located in proximity to V7 of MCoTI-II are displayed as sticks and spheres. 


\section{Modeling of the trypsin MCoTI-II complex}

Starting structures were the NMR structure of McoTI-II (PDB entry 1HA9; Heitz et al., 2001) and the crystal structure of a complex of EETI-II with porcine trypsin (PDB entry $1 \mathrm{H} 9 \mathrm{H}$; Krätzner et al., 2005). MCoTI-II was superimposed onto the EETI-II structure by pairwise superposition of structurally equivalent heavy atom pairs of all six cysteine residues using PYMOL (Delano, 2002).

\section{Acknowledgments}

We would like to thank Laurent Chiche for helpful discussions and Pham Thi Tran Chau, Jean-François Hernandez and Annie Heitz for samples of MCoTI-I, -II and -III. We also thank Holm Frauendorf and Györgyi Udvarnoki for ESI-MS, and Sabine Streicher for enzyme kinetics. This work was supported by the Deutsche Forschungsgemeinschaft through Sonderforschungsbereich 416 .

\section{References}

Albericio, F., Annis, I., Royo, M., and Barany, G. (2000). Preparation and handling of peptides containing methionine and cysteine. In: Fmoc Solid Phase Peptide Synthesis. A Practical Approach, W.C. Chan and P.D. White, eds. (New York, USA: Oxford University Press), pp. 81-91.

Avrutina, O., Schmoldt, H.U., Kolmar, H., and Diederichsen, U. (2004). Fmoc-assisted synthesis of a 29-residue cystine-knot trypsin inhibitor containing a guaninyl amino acid at the P1position. Eur. J. Org. Chem. 2004, 4931-4935.

Barry, D.G., Daly, N.L., Clark, R.J., Sando, L., and Craik, D.J. (2003). Linearization of a naturally occurring circular protein maintains structure but eliminates hemolytic activity. Biochemistry 42, 6688-6695.

Bieth, J.G. (1980). Pathophysiological interpretation of kinetic constants of protease inhibitors. Bull. Eur. Physiopathol. Respir. 16 (Suppl.), 183-197.

Bode, W., Greyling, H.J., Huber, R., Otlewski, J., and Wilusz, T. (1989). The refined $2.0 \AA$ X-ray crystal structure of the complex formed between bovine $\beta$-trypsin and CMTI-I, a trypsin inhibitor from squash seeds (Cucurbita maxima). Topological similarity of the squash seed inhibitors with the carboxypeptidase A inhibitor from potatoes. FEBS Lett. 242, 285-292.

Chase, T. Jr. and Shaw, E. (1970). Titration of trypsin, plasmin, and thrombin with $p$-nitrophenyl- $p^{\prime}$-guanidinobenzoate $\mathrm{HCl}$. In: Methods in Enzymology, G.E. Perlmann, and L. Lorand, eds. (New York, USA: Academic Press), pp. 20-27.

Chiche, L., Heitz, A., Gelly, J.C., Gracy, J., Chau, P.T., Ha, P.T., Hernandez, J.F., and Le-Nguyen, D. (2004). Squash inhibitors: from structural motifs to macrocyclic knottins. Curr. Protein Pept. Sci. 5, 341-349.

Colgrave, M.L. and Craik, D.J. (2004). Thermal, chemical, and enzymatic stability of the cyclotide kalata B1: the importance of the cyclic cystine knot. Biochemistry 43, 5965-5975.

Craik, D.J. (2001). Plant cyclotides: circular, knotted peptide toxins. Toxicon 39, 1809-1813.

Daly, N.L., Love, S., Alewood, P.F., and Craik, D.J. (1999). Chemical synthesis and folding pathways of large cyclic polypeptides: studies of the cystine knot polypeptide kalata B1. Biochemistry 38, 10606-10614.
Delano, W.L. (2002). The PyMOL Molecular Graphics System on World Wide Web, www.pymol.org (San Carlos, USA: DeLano Scientific).

Domingo, G.J., Leatherbarrow, R.J., Freeman, N., Patel, S., and Weir, M. (1995). Synthesis of a mixture of cyclic peptides based on the Bowman-Birk reactive site loop to screen for serine protease inhibitors. Int. J. Pept. Protein Res. 46, 7987.

Favel, A., Mattras, H., Coletti-Previero, M.A., Zwilling, R., Robinson, E.A., and Castro, B. (1989). Protease inhibitors from Ecballium elaterium seeds. Int. J. Pept. Protein Res. 33, 202-208.

Felizmenio-Quimio, M.E., Daly, N.L., and Craik, D.J. (2001). Circular proteins in plants: solution structure of a novel macrocyclic trypsin inhibitor from Momordica cochinchinensis. J. Biol. Chem. 276, 22875-22882.

Gustafson, K.R., McKee, T.C., and Bokesch, H.R. (2004). AntiHIV cyclotides. Curr. Protein Pept. Sci. 5, 331-340.

Heitz, A., Hernandez, J.F., Gagnon, J., Hong, T.T., Pham, T.T., Nguyen, T.M., Le-Nguyen, D., and Chiche, L. (2001). Solution structure of the squash trypsin inhibitor MCoTI-II. A new family for cyclic knottins. Biochemistry 40, 7973-7983.

Hernandez, J.F., Gagnon, J., Chiche, L., Nguyen, T.M., Andrieu, J.P., Heitz, A., Trinh Hong, T., Pham, T.T., and Le Nguyen, D. (2000). Squash trypsin inhibitors from Momordica cochinchinensis exhibit an atypical macrocyclic structure. Biochemistry $39,5722-5730$.

Jennings, C., West, J., Waine, C., Craik, D., and Anderson, M. (2001). Biosynthesis and insecticidal properties of plant cyclotides: the cyclic knotted proteins from Oldenlandia affinis. Proc. Natl. Acad. Sci. USA 98, 10614-10619.

Kokko, K.P., Arrigoni, C.E., and Dix, T.A. (2001). Selectivity enhancement induced by substitution of non-natural analogues of arginine and lysine in arginine-based thrombin inhibitors. Bioorg. Med. Chem. Lett. 11, 1947-1950.

Krätzner, R., Debreczeni, J.É., Pape, T., Schneider, T.R., Wentzel, A., Kolmar, H., Sheldrick, G.M., and Uson, I. (2005). Structure of Ecballium elaterium trypsin inhibitor II (EETI-II): a rigid molecular scaffold. Acta Cryst. D61, 1255-1262.

Lorenz, K.B. and Diederichsen, U. (2003). Nucleo amino acids as arginine mimetics in cyclic peptides. Lett. Pept. Sci. 10, 111-117.

McBride, J.D. and Leatherbarrow, R.J. (2001). Synthetic peptide mimics of the Bowman-Birk inhibitor protein. Curr. Med. Chem. 8, 909-917.

Morrison, J.F. (1969). Kinetics of the reversible inhibition of enzyme-catalysed reactions by tight-binding inhibitors. Biochim. Biophys. Acta 185, 269-286.

Schechter, I. and Berger, A. (1967). On the size of the active site in proteases. I. Papain. Biochem. Biophys. Res. Commun. $27,157-162$

Tam, J.P., Lu, Y.A., Yang, J.L., and Chiu, K.W. (1999). An unusual structural motif of antimicrobial peptides containing end-toend macrocycle and cystine-knot disulfides. Proc. Natl. Acad. Sci. USA 96, 8913-8918.

Trabi, M. and Craik, D.J. (2002). Circular proteins - no end in sight. Trends Biochem. Sci. 27, 132-138.

Witherup, K.M., Bogusky, M.J., Anderson, P.S., Ramjit, H., Ransom, R.W., Wood, T., and Sardana, M. (1994). Cyclopsychotride $\mathrm{A}$, a biologically active, 31-residue cyclic peptide isolated from Psychotria longipes. J. Nat. Prod. 57, 16191625.

Received June 13, 2005; accepted September 14, 2005 